\title{
ARTICLE
}

Epidemiology and Population Health

\section{Does weight loss reduce the incidence of total knee and hip replacement for osteoarthritis?-A prospective cohort study among middle-aged and older adults with overweight or obesity}

\author{
Xingzhong Jin $\mathbb{1}^{1,2,3} \cdot$ Alice A. Gibson ${ }^{4} \cdot$ Joanne Gale $^{5} \cdot$ Francisco Schneuer $^{6} \cdot$ Ding Ding $\mathbb{D}^{5} \cdot$ Lyn $^{\text {March }}{ }^{2}$. \\ Amanda Sainsbury $\left(\mathbb{D}^{7} \cdot\right.$ Natasha Nassar $^{5}$
}

Received: 30 September 2020 / Revised: 17 March 2021 / Accepted: 21 April 2021 / Published online: 15 May 2021

(c) The Author(s), under exclusive licence to Springer Nature Limited 2021. This article is published with open access

\begin{abstract}
Objective This study aims to investigate the association between weight change and total knee or hip replacement (TKR or THR) for OA among middle-aged and older adults with overweight or obesity.

Method Weight data were collected in 2006-2009 and in 2010 from the 45 and Up Study-a population-based cohort aged $\geq 45$ years in New South Wales, Australia. Participants were included if they had a baseline body mass index (BMI) $\geq 25 \mathrm{~kg} /$ $\mathrm{m}^{2}$ and no history of TKR or THR. Weight change was categorised into four groups: $>7.5 \%$ loss; $>5-7.5 \%$ loss; stable $(\leq 5 \%$ change) and $>5 \%$ gain. Hospital admission data were linked to identify TKR and THR for OA, and multivariable Cox regression was used to assess risk of TKR and THR.

Results Of 23,916 participants, 2139 lost $>7.5 \%$ weight, 1655 lost 5-7.5\% weight, and 4430 gained $>5 \%$ weight. Over 5.2 years, $1009(4.2 \%)$ underwent TKR and 483 (2.0\%) THR. Compared to weight-stable, weight loss of $>7.5 \%$ was associated with reduced risk of TKR after adjusting for age, sex, BMI, socioeconomic and lifestyle factors (hazard ratio 0.69, 95\%CI 0.54-0.87), but had no association with THR. Weight loss of 5-7.5\% was not associated with altered risk of either TKR or THR. Weight gain was associated with increased risk of THR after adjusting for confounders, but not TKR.

Conclusion This study suggests that a weight loss target $>7.5 \%$ is required to reduce the risk of TKR in adults with overweight or obesity. Weight gain should be avoided as it increases the risk of THR.
\end{abstract}

\section{Introduction}

Osteoarthritis (OA) is a public health problem and was ranked as the 11th highest contributor (out of 310 diseases) to global disability in 2015 [1]. Large weight-bearing joints,

Supplementary information The online version contains supplementary material available at https://doi.org/10.1038/s41366021-00832-3.

Xingzhong Jin

xingzhong.jin@unsw.edu.au

1 Centre for Big Data Research in Health, The University of New South Wales, Sydney, Australia

2 Institute of Bone and Joint Research, Kolling Institute, The University of Sydney, Sydney, Australia

3 The Boden Collaboration for Obesity, Nutrition, Exercise \& Eating Disorders, , The University of Sydney, Sydney, Australia

4 Menzies Centre for Health Policy, Sydney School of Public notably the knee and hip, are usually affected by the disease in people aged 45 and over [2]. Presently, there are no approved pharmacotherapies that prevent or halt the progression of OA. For severe knee or hip OA, the 'last resort' treatments are total knee or hip replacement surgery (TKR or THR). The global economic burden of TKR and THR due to $\mathrm{OA}$ is high [3], and is predicted to increase substantially in the next two decades in many countries, including the United States [4], United Kingdom [5], Canada [6], Sweden [7] and New Zealand [8]. In Australia, the healthcare costs

Health, Faculty of Medicine and Health, The University of Sydney, Sydney, Australia

5 Sydney School of Public Health, The University of Sydney, Sydney, Australia

6 Child Population and Translational Health, The Children's Hospital at Westmead Clinical School, The University of Sydney, Sydney, Australia

7 School of Human Sciences, The University of Western Australia, Perth, Australia 
for OA were over $\$ 2.1$ billion in 2015 , and $77 \%$ of these costs were attributable to admitted hospital costs for TKR or THR for OA [9]. By the year 2030, the incidence of TKR and THR in Australia is expected to rise by $276 \%$ and $208 \%$, respectively [10]. Similar projections were also reported for other countries $[4,5]$ due to an aging world population and the increasing prevalence of obesity [11].

Obesity is a well-recognised risk factor for the development and progression of OA, and likely contributes to the need for total joint replacement [12]. A recent meta-analysis found that people with obesity had a 4.6-fold greater risk of developing OA compared to people with healthy weight [13]. People with excess weight are more likely to progress to an advanced stage of $\mathrm{OA}$, with estimates suggesting that 80-95\% of people needing a total joint replacement also had overweight or obesity [14, 15]. Considering the strong link between obesity and increased risk of $\mathrm{OA}$ as well as total joint replacement, all clinical guidelines for OA management around the world recommend weight loss as a core strategy for all OA patients with overweight and obesity [16-20]. However, few studies have assessed and quantified the clinical benefits of weight loss for OA. A systematic review and meta-analysis of four randomised controlled trials showed a statistically significant effect of weight loss to reduce pain and disability in people with knee OA, but it was unclear whether these statistically significant changes were of sufficient magnitude to be clinically significant [21]. Indeed, the meta-analysis suggested that a loss of $5 \%$ of initial weight within a 20-week period is required to obtain moderate symptomatic relief [21]. In contrast to these studies on knee $\mathrm{OA}$, there are no studies investigating the specific effects of weight loss on hip OA. More importantly, for both knee and hip OA, it remains unclear whether weight loss reduces the risk for TKR and THR in the long term. Therefore, the objective of this longitudinal study was to investigate the association of weight loss recommended by current guidelines and the risk of TKR and THR due to OA among middle-aged and older adults with overweight or obesity.

\section{Method}

\section{Study design and data sources}

This study used data from the Sax Institute's 45 and Up Study, which is a large longitudinal study of healthy ageing in the general population living in New South Wales (NSW), Australia [22]. The research protocol has been published in detail previously [23], and is described briefly here. Prospective participants were randomly sampled from the enrolment database of the Department of Human Services, which provides near-complete coverage of the Australian population. People living in rural areas and those

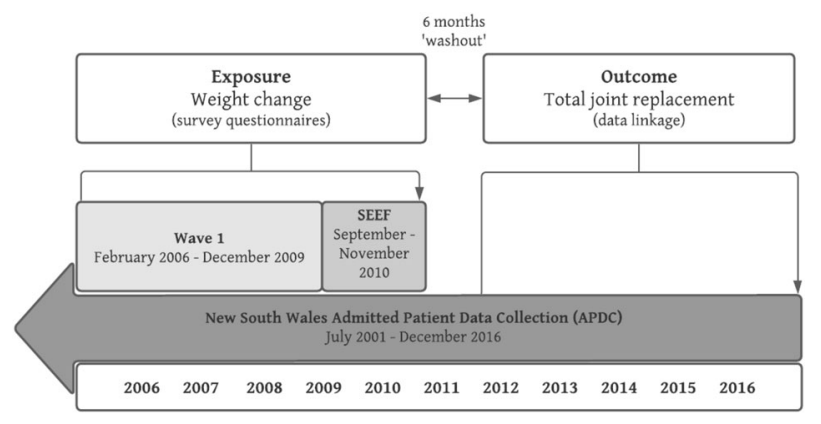

Fig. 1 Study design schema.

aged 80 years and over were oversampled to provide clear statistical pictures of these groups. Baseline data (Wave 1) were collected via self-report surveys between February 2006 and December 2009 from 267,153 individuals aged $\geq 45$ years (Fig. 1). These participants provided informed consent for subsequent follow-up surveys and access to their data from administrative health records via data linkage. Following baseline survey, the first 100,000 participants were contacted for a second survey in September to November 2010, as part of a separate study, the Social Economic and Environmental Factors (SEEF) Study, and were included in our study so we could measure a longitudinal change in weight (Fig. 1).

For the current study, the SEEF study participants were followed up for outcome data collection via probabilistic data linkage to their corresponding administrative hospital and death records. These were obtained from the NSW Admitted Patient Data Collection (APDC, July 2001 to December 2016) and the NSW Registry of Births, Death and Marriages (January 2006 to December 2016), respectively (Fig. 1). Data linkage was conducted by the NSW Centre for Health Record Linkage.

\section{Ethics committee approval}

Ethics approval for the 45 and Up study was granted by the University of NSW Human Research Ethics Committee, and approval for the current study was obtained from the NSW Population Health Services Research Ethics Committee.

\section{Patient and public involvement (PPI)}

We did not directly include PPI in this study, but the database used in the study was developed with PPI and is updated by a committee that includes patient representatives.

\section{Study exposure, outcomes and confounders}

Our exposure of interest was the change in weight from the baseline (Wave 1) to follow-up (SEEF) surveys. The weight 
data were self-reported at both timepoints. Weight change was categorised into four groups: weight loss $>7.5 \%$; weight loss $>5-7.5 \%$; weight-stable ( $\leq 5 \%$ change), and weight gain $>5 \%$. We used $5 \%$ and $7.5 \%$ as cut-offs for weight loss because current guidelines from the Osteoarthritis Research Society International [16] and the Royal Australian College of General Practitioners [20] recommend a weight loss target of $5 \%$ and $5-7.5 \%$ of initial weight, respectively, for effective management of $\mathrm{OA}$ in people with overweight or obesity.

Study outcomes were defined as the incidence of TKR or THR for OA and determined using the linked hospital admission records from the NSW Admitted Patient Data Collection (APDC, Fig. 1). The APDC is a statutory statewide, administrative data collection of all public and private hospital in-patient admissions in NSW, Australia. The APDC data were linked to the 45 and Up Study using probabilistic matching conducted by the NSW Centre for Health Record Linkage (CHeReL) with a false positive rate of $0.5 \%$. Cases of TKR and THR were identified according to the codes used by the Australian National Joint Replacement Registry [24], which include a procedure code for TKR or THR based on the Australian Classification of Healthcare Interventions (ACHI) procedural codes, together with a primary diagnosis code for knee OA or hip OA based on the International Classification of Diseases, 10th Revision, Australian Modification (ICD10-AM). If a participant had multiple knee (or hip) replacements during the followup, the first replacement surgery of the knee (or hip) was used to defined as an incidence.

We selected a list of confounders based on background knowledge and prior literature evidence. Potential confounders selected for this study were age, sex, body mass index (BMI), education level [25], private health insurance status [26], smoking status [27], physical activity level [28], and self-reported OA status ("In the last month have you been treated for osteoarthritis"). Information of these confounders were based on self-reported responses at the baseline survey (Wave 1). BMI was derived from body weight in kilograms divided by the square of the body height in metres. Physical activity level was assessed using the Active Australia Survey [29]. The number of minutes spent on physical activity per week was calculated as the sum of the number of minutes spent on three types of activity, namely walking (generally considered as low-intensity), moderate-intensity, and vigorous-intensity physical activity, with the minutes of vigorous-intensity physical activity weighted by 2 according to the Active Australia Protocol [29]. Based on the minutes of physical activity per week, participants were classified into one of three physical activity levels: $<150 \mathrm{~min}$ (inactive or insufficiently active); 150 to 299 min (sufficiently active); or $\geq 300 \mathrm{~min}$ (highly active). This classification is similar to the Australian's Physical
Activity and Sedentary Behaviour Guidelines [30], except that minutes of walking is not included in the government guidelines.

\section{Study participants}

Participants were included in the present study if they had: valid weight data collected in both the 45 and Up Study (baseline Wave 1) and SEEF Study (follow-up), a BMI $\geq$ $25 \mathrm{~kg} / \mathrm{m}^{2}$ and no self-reported diagnosis of cancer at baseline (because cancer is often associated with unintentional weight loss). Participants who had a TKR (or THR) before 6 months following the completion of the SEEF Study survey were further excluded from the TKR (or THR) analysis sample. This exclusion procedure was conducted because patients undergoing elective TKR (or THR) are routinely advised to lose weight 6 months prior to surgery if they have overweight or obesity [31], and including these participants in our study would bias the analysis toward a positive association between weight loss and total joint replacement.

\section{Statistical analysis}

Means and standard deviations (for continuous variables) and percentages (for categorical variables) were used to describe the baseline characteristics of participants, grouped by weight change categories. The associations between weight change and the risk for TKR and THR were each analysed using Cox proportional hazards regression models and hazard ratios (HRs), and their $95 \%$ confidence intervals (CIs) were calculated. Weight-stable ( $\leq 5 \%$ change) was used as the reference group for comparison. Following univariable analysis, multivariable analyses were conducted, first adjusting for age, sex and baseline BMI (Model 1 ), and then additionally adjusting for education level, private health insurance status, smoking status, physical activity level, and OA status (Model 2). Given the impact OA status may have on findings, we examined the potential interaction between $\mathrm{OA}$ status and weight change. We also conducted sensitivity analyses using percentage of weight loss as continuous variable to explore the linear relationship with the risk of TKR and THR. All analyses were performed using SAS software version 9.4 (SAS Institute Inc., Cary, North Carolina), and statistical significance was defined as a two-tailed $p$-value $<0.05$.

\section{Results}

A flowchart of participants in this study is presented in Fig. 2. A total of 60,336 participants responded to the follow-up survey (response rate 60.3\%). The characteristics of the 
Fig. 2 Study sampling flowchart.

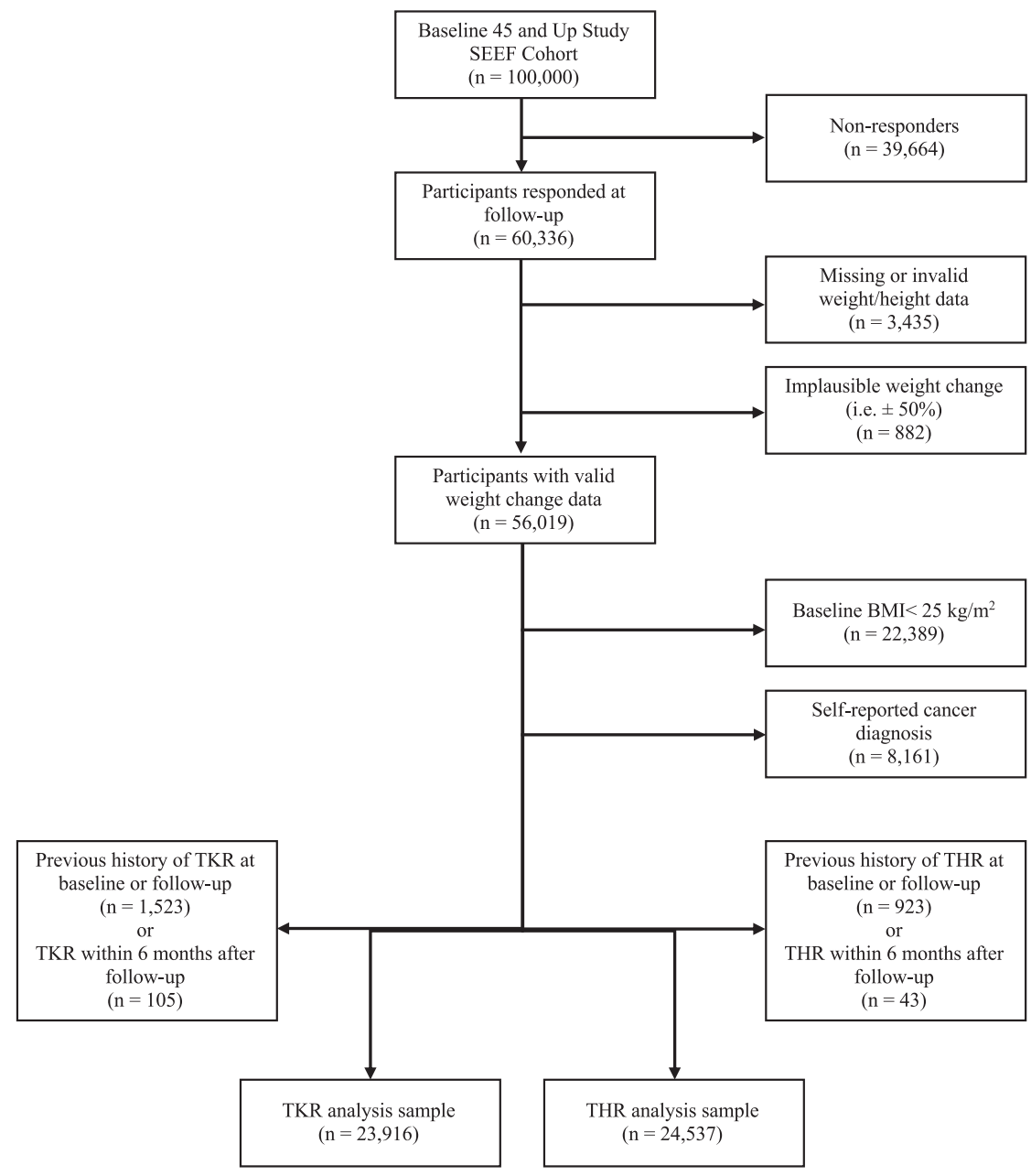

participants who responded were comparable to the original 45 and Up Study cohort in terms of sex, BMI and OA status (Supplementary Table 1). After exclusion of ineligible participants, a total of 25,469 participants were available for this study (Fig. 2). Of these, 23,916 and 24,537 participants were included in the analysis of TKR and THR for OA, respectively. Table 1 presents the baseline characteristics of the cohort included in the analysis of TKR for OA. The baseline characteristics of the cohort included in the analysis of THR for OA were similar (Supplementary Table 2). The four weight change groups were similar in terms of age, sex, BMI and other potential confounders selected for this study.

\section{Total knee replacement for osteoarthritis}

For the analyses of TKR for OA, most participants $(65.6 \%)$ maintained a stable weight $(\leq 5 \%$ change), while 2,139 (8.9\%) lost $>7.5 \%$ weight, 1,655 (6.9\%) lost $>5-7.5 \%$ weight, and 4,430 (18.5\%) gained $>5 \%$ weight (Table 1 ). The mean follow-up time for the TKR analysis was 5.2 years (range 4.7-6.0 years), and a total of 1,009 (4.2\%) underwent TKR for OA during this follow-up period.
Compared to those with a stable weight, individuals with weight loss $>7.5 \%$ had a reduced risk of TKR (HR 0.69, 95\%CI 0.54-0.87) after adjusting for age, sex and baseline BMI (Multivariable Model 1, Fig. 3). There was little change in the results after further adjusting for education level, private health insurance status, smoking status, physical activity level and OA status (HR 0.68, 95\%CI 0.53-0.87, Multivariable Model 2, Fig. 3). Neither $>5-7.5 \%$ weight loss nor $>5 \%$ weight gain was significantly associated with altered risk of TKR. There was also no significant interaction between $\mathrm{OA}$ status and weight change $(p=0.70)$. In the sensitivity analysis, a linear association was observed between percentage of weight loss and risk of TKR (HR 1.02, 95\%CI 1.01-1.03). In other words, for every $1 \%$ reduction in weight, there was a $2 \%$ reduction in risk of TKR.

\section{Total hip replacement for osteoarthritis}

The mean follow-up time between exposure and outcome data collection for this cohort was 5.2 years (range 4.7-6.0 years), and 483 (2.0\%) had THR for OA during this follow- 
Table 1 Baseline characteristics and knee replacement rate of participants in the total knee replacement for osteoarthritis analysis.

\begin{tabular}{|c|c|c|c|c|c|}
\hline & \multicolumn{4}{|c|}{ Weight change from baseline to follow-up } & \multirow[t]{2}{*}{ Total } \\
\hline & Loss $>7.5 \%$ & Loss $5-7.5 \%$ & Stable & Gain $>5 \%$ & \\
\hline$n$ & 2139 & 1655 & 15,692 & 4430 & 23,916 \\
\hline Age (years) & $61.5 \pm 10.7$ & $62 \pm 10.2$ & $60.6 \pm 9.4$ & $57.7 \pm 8.4$ & $60.2 \pm 9.5$ \\
\hline Female $(\%)$ & $1260(58.9)$ & $837(50.6)$ & $7127(45.4)$ & $2605(58.8)$ & $11,829(49.5)$ \\
\hline Body mass index $\left(\mathrm{kg} / \mathrm{m}^{2}\right)$ & $31.3 \pm 5.8$ & $29.7 \pm 4.3$ & $29.2 \pm 3.9$ & $29.5 \pm 4.2$ & $29.5 \pm 4.3$ \\
\hline $\begin{array}{l}\text { Treated for } \mathrm{OA} \text { in the last } \\
\text { month }(\%)\end{array}$ & $331(15.5)$ & $232(14.0)$ & $1831(11.7)$ & $544(12.3)$ & $2938(12.3)$ \\
\hline \multicolumn{6}{|l|}{ Education } \\
\hline $\begin{array}{l}\text { Up to School or Intermediate } \\
\text { Certificate }\end{array}$ & $750(35.1)$ & $518(31.3)$ & $4614(29.4)$ & $1406(31.7)$ & $7288(30.5)$ \\
\hline Higher School to Diploma & $846(39.6)$ & $718(43.4)$ & $6806(43.4)$ & $1929(43.5)$ & $10,299(43.1)$ \\
\hline Degree of higher & $519(24.3)$ & $397(24.0)$ & $4105(26.2)$ & $1043(23.5)$ & $6064(25.4)$ \\
\hline $\begin{array}{l}\text { Have private health } \\
\text { insurance }(\%)\end{array}$ & $1346(62.9)$ & $1085(65.6)$ & $10,858(69.2)$ & $2897(65.4)$ & $16,186(67.7)$ \\
\hline \multicolumn{6}{|l|}{ Physical activity level (\%) } \\
\hline$<150$ min per week & $518(24.2)$ & $331(20.0)$ & $2978(19.0)$ & $919(20.7)$ & $4746(19.8)$ \\
\hline 150-299 min per week & $358(16.7)$ & $264(16.0)$ & $2667(17.0)$ & $763(17.2)$ & $4052(16.9)$ \\
\hline $300+$ minutes per week & $1193(55.8)$ & 1003 (60.6) & 9699 (61.8) & $2657(60.0)$ & $14,552(60.8)$ \\
\hline \multicolumn{6}{|l|}{ Smoking status (\%) } \\
\hline Non-smoker & $1197(56.0)$ & 903 (54.6) & $9123(58.1)$ & $2388(53.9)$ & $13,611(56.9)$ \\
\hline Ex-smoker & 786 (36.7) & 667 (40.3) & $5837(37.2)$ & $1649(37.2)$ & 8939 (37.4) \\
\hline Current smoker & $156(7.3)$ & $85(5.1)$ & $731(4.7)$ & $393(8.9)$ & $1365(5.7)$ \\
\hline
\end{tabular}

$O A$, osteoarthritis.

Numbers may not add up to totals due to missing data.

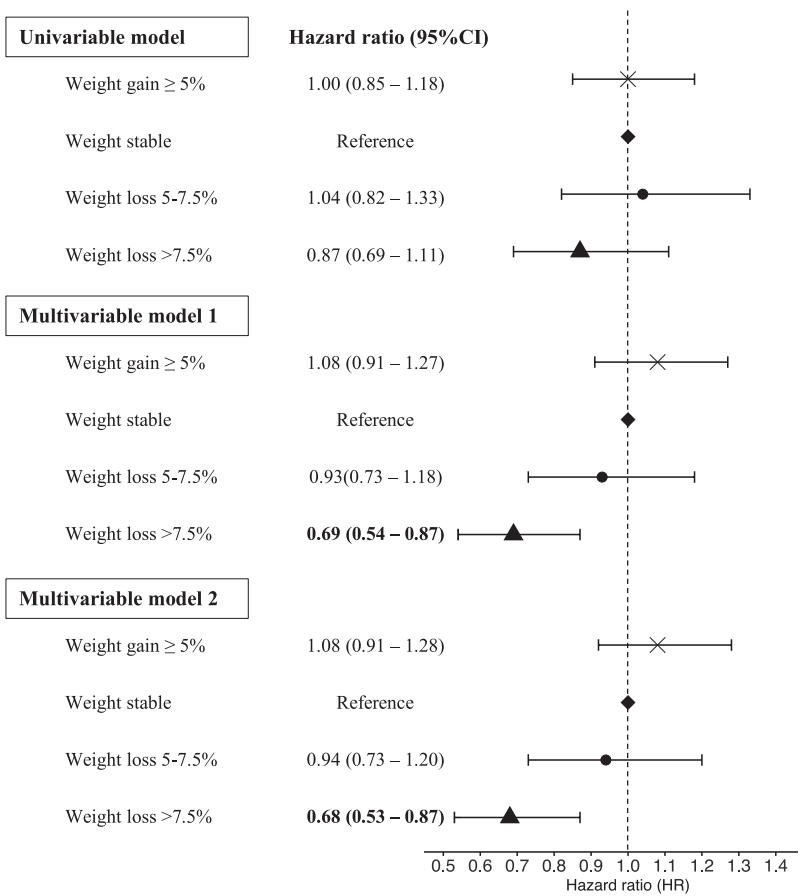

Fig. 3 Association between weight change and incidence of total knee replacement. up period. Similar to the TKR analysis, the majority of participants $(65.5 \%)$ maintained a stable weight $(\leq 5 \%$ change), while $2,222(9.1 \%)$ lost $>7.5 \%$ weight, 1,692 $(6.9 \%)$ lost $>5-7.5 \%$ weight, and 4,562 (18.6\%) gained $>5 \%$ weight. In contrast to TKR, those who lost $>7.5 \%$ weight did not appear to have a significantly increased risk of THR for OA compared to the weight-stable reference group (Fig. 4). However, those who gained $>5 \%$ weight had an increased risk of THR for OA, with an HR of 1.25 (95\% CI 1.00-1.56) (Fig. 4). The association was borderline significant, but became stronger after adjusting for confounders (Model 1 and Model 2, Fig. 4). There was no interaction between OA status and weight change $(p>$ $0.05)$. The linear association between percentage of weight change and risk of THR was not statistically significant (HR $1.01,95 \%$ CI 1.00-1.03).

\section{Discussion}

This study provides circumstantial evidence that middleaged and older adults with overweight or obesity who lost $>7.5 \%$ of their initial weight had reduced risk of TKR for 


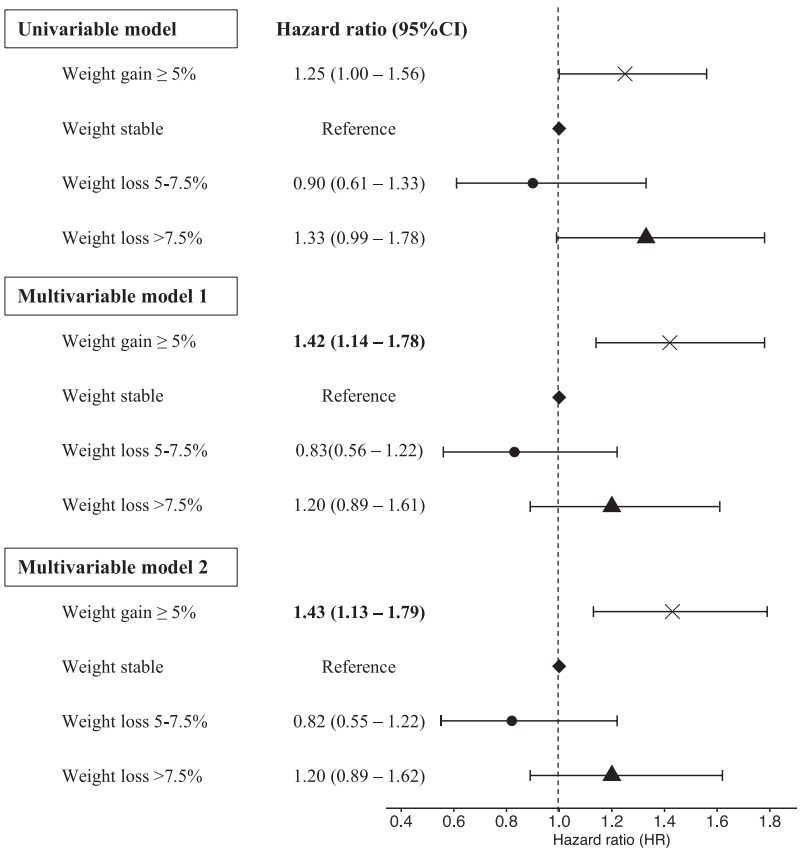

Fig. 4 Association between weight change and incidence of total hip replacement.

OA compared to those with a stable weight ( $\leq 5 \%$ change). Neither losing $>5-7.5 \%$ of weight nor gaining $>5 \%$ weight significantly impacted the risk of TKR. Although losing weight was not associated with the risk of THR, gaining weight increased the risk of THR for OA.

Our results extend previous research which highlighted the benefits of weight loss on knee OA symptoms. Evidence from a systematic review and meta-analysis suggested that weight loss was likely to reduce symptoms of OA and improve joint function in individuals with overweight and knee OA [21]. The effect of weight loss on symptom reduction in knee OA appears to have a dose-response relationship [32]. Specifically, weight loss of at least 7.7\% was required to achieve a clinically meaningful improvement in pain and physical function in patients with knee OA and concurrent overweight or obesity [32]. The findings from our analyses show a similar trend between weight loss and reduction in TKR for OA, and suggest that a weight loss of at least $7.5 \%$ is required to reduce TKR for OA. Furthermore, we conducted an exploratory analysis stratified by overweight and obesity subgroups. The results showed that while the association between weight loss $>7.5 \%$ and reduced TKR risk remained and was statistically significant in the overweight subgroup, there was no association in the obesity subgroup, suggesting a higher percentage of weight loss is required for the obesity group.

It is worth noting that the lack of statistical significance in the association between weight gain and TKR could be due to a statistical 'ceiling effect'-i.e., the variance of weight change was no longer measurable when participants were grouped into the weight gain category. To explore this ceiling effect of weight gain, we conducted a subgroup analysis that used percentage weight loss as a continuous variable and examine the linear association separately in participants who lost and gained weight. The analysis showed a significant linear association between increasing percentage of weight change and TKR in both weight gain (HR 1.03, 95\%CI 1.01-1.04) and weight-loss (HR 1.04, 95\%CI 1.02-1.06) subgroups. Although the elevated HR for TKR in the weight gain $>5 \%$ group in our primary analysis did not reach a statistical significance when comparing to the weight stable group, people with overweight or obesity should still avoid further weight gain for the benefits of reducing the risk of cardiovascular comorbidities and diabetes [33].

The current study addresses the knowledge gap as to whether or not weight loss slows the progression of knee OA that leads to joint replacement surgery [34]. One study in a cohort of people with obesity showed that weight loss was associated with reduced medial cartilage volume loss in the knees over 2.3 years, suggesting a disease-modifying effect of weight loss on knee joint structure [35]. Data from the Osteoarthritis Initiative also found that an appreciable weight loss ( $>10 \%$ of initial weight) slowed knee cartilage degeneration in people with overweight and obesity over a period of 4 years [36]. In contrast, a randomised controlled trial reported that no differences were observed in cartilage loss, bone marrow lesion or synovitis over 1.5 years, between people with OA who lost $\geq 10 \%$ of their initial weight compared to those who did not [37]. Similarly, in a more recent study among individuals with severe obesity undergoing either bariatric surgery, pharmacotherapy or other non-surgical medical interventions for weight loss, participants who lost $\geq 20 \%$ of their initial body weight had a rapid reduction in pain from knee OA over 1 year, but this was not accompanied by structural changes in the knee compared to those who lost $<20 \%$ of their initial weight [38]. The results from our study address this knowledge gap by showing the long-term benefit of weight loss $>7.5 \%$ in reducing progression to TKR for OA in individuals with overweight or obesity.

Although weight loss is recommended for patients with hip OA by most OA guidelines [17, 18, 20], evidence of its benefits on hip OA symptoms and THR is lacking. In the latest OARSI guidelines for non-surgical management for hip OA, dietary weight management was not recommended 'because of lack of direct evidence for its effectiveness specifically for symptoms of hip OA' [39]. One prospective cohort study reported that weight loss in combination with exercise could reduce pain from hip OA and increase hip physical function after 8 months [40], while another prospective cohort study reported that overweight was not a determinant of the onset or progression of hip radiographic 
OA [41]. Our results were in agreement with-and extend - the latter study, suggesting that weight loss was not associated with reduced risk of THR for OA. The null association may be a result of the hip joint being less sensitive to obesity and weight change than the knee joint [41, 42]. It is possible that the reduction in mechanical loading of the joint resulting from weight loss is different in the knee and the hip. The knee and the hip have different anatomy: the knee joint is a hinge joint, whereas the hip is a ball-and-socket joint. Changes in force on a misaligned hinge joint (e.g., an OA-affected knee joint) may be magnified by two to three times compared with a normal hinge joint, due to the small area that the forces act on [41]. In contrast, a misaligned ball-and-socket joint (e.g., an OAaffected hip joint) might be less sensitive to changes in force, as they are distributed over a larger area compared to the knee. These findings do not negate the notion that individuals with overweight or obesity should avoid further weight gain, which was shown to significantly increase the risk of THR for OA in the present study.

\section{Strengths and limitations}

To our knowledge, the present study is the first prospective cohort study to investigate the association of weight loss on the reduction in TKR and THR for OA. A major strength of our study design is that, instead of analysing a cross-sectional correlation between current weight and joint replacement surgery, we observed the long-term incidence of TKR and THR for OA after weight change had occurred. Other strengths of this study include the large sample size from a population-based cohort (over 60,000 people), and the use of data linkage to state-wide hospital and death records to ascertain outcomes. We also acknowledge that there are limitations inherent to this study. One limitation is that OA at baseline was defined by self-reported treatment for OA, and we did not have information on whether OA affects the knees, the hips or both, which may have introduced recall bias or misclassification bias [43]. Therefore, we included both OA and non-OA population in the main analysis in an effort to mitigate the impact of these biases. This also extends the generalisability of the results to a broader population with overweight or obesity. Another limitation is that our analyses were not able to explore the confounding effect of important risk factors for receiving TKR and THR, such as the previous history of joint injury, or disease severity of OA measured by $\mathrm{X}$-ray and joint pain, because these data were not recorded in this population cohort study. Also, our data did not record details about how the weight loss was achieved (e.g. via dietary treatment, physical exercise, or bariatric surgery), it is possible that different means of weight loss may influence the risk of TKR and THR differently due to various physiological mechanisms. Lastly, we were not able to identify the side of the body on which the TKR or THR was performed, because the ICD10-AM codes used to report TKR or THR do not specify sidedness. As a result, we excluded participants who self-reported having had any previous TKR or THR at the start of follow-up, in order to reduce contamination by repeated TKR or THR. However, we do not see any reason why the observed effects of weight loss would not also be applicable to the opposite side of the body, for people who have already had a TKR or THR on one side of the body. National joint replacement registries, such as the Australian Orthopaedic Association National Joint Replacement Registry (AOANJRR), usually have laterality information recorded. Future studies that have the data linkage capacity with a national joint replacement registry will be able to confirm the effects of weight loss on the risk of contralateral joint replacement in people with one side of the joints replaced.

Even though there are very few contraindications to TKR and THR and the mortality rates following the procedures have decreased substantially over recent decades, there are serious adverse events associated with both of these procedures, including sepsis, pulmonary embolism and cardiac complications [44]. Besides, approximately $20 \%$ and $9 \%$ of patients experience persistent joint pain 12 months after TKR and THR, respectively [45]. Therefore, joint replacement treatment should be reserved as the last treatment option for those with end-stage OA when conservative management has failed [20]. Setting an evidence-based weight loss target is important for effectively managing knee and hip OA to reduce or delay the need for joint replacement surgery. While weight loss is recommended as a core management strategy in OA clinical management guidelines around the world, few of them highlights a necessity for weight loss of over $7.5 \%$. For example, the Osteoarthritis Research Society International recommends a weight loss of 5\% to be efficacious [16], while the Royal Australian College of General Practitioners recommend a minimum weight loss target of 5-7.5\% for all patients with OA and overweight or obesity [20]. The present study suggests a weight loss target of $>7.5 \%$ to reduce the risk of TKR for OA. Future studies are needed to address the limitations of this study. In particular, the weight loss target $>7.5 \%$ should be further confirmed in clinical trials or in long-term epidemiology studies in an OA population, such as the Osteoarthritis Initiative [46].

\section{Conclusion}

A weight loss of $>7.5 \%$ is associated with a reduced risk of TKR for OA among middle-aged and older adults with overweight or obesity. Further weight gain should be avoided to prevent an increased risk of THR for OA. 


\section{Data sharing statement}

This research was completed using data collected from the Sax Institute's 45 and Up Study. Requests for access to data should be addressed to the corresponding author or the Sax Institute (http://www.saxinstitute.org.au/).

Acknowledgements The 45 and Up Study is managed by the Sax Institute in collaboration with its major partner, Cancer Council NSW, as well as the Cancer Council NSW partners: the National Heart Foundation of Australia (NSW Division); the NSW Ministry of Health; the NSW Government Family \& Community ServicesAgeing, Carers and the Disability Council NSW; and the Australian Red Cross Blood Service. We thank the many thousands of people participating in the 45 and Up Study. We also acknowledge the NSW Centre for Health Record Linkage for data linkage.

Author contributions X.J., A.A.G., D.D., L.M., A.S. and N.N. contributed to the conception and design of the research. A.A.G., J.G., F. S., D.D. and N.N. contributed to the acquisition and analysis of data. X.J., L.M., A.S. and N.N. contributed to the interpretation of the results. X.J. drafted the manuscript and all authors contributed to the critical revision of the written manuscript for publication.

Funding X.J. is funded by an Early Career Fellowship (\#1143022) from the National Health and Medical Research Council (NHMRC) of Australia. DD is funded by a Heart Foundation Future Leader Fellowship (\#101234). A.S. is funded by a Senior Research Fellowship (\#1135897) from the NHMRC of Australia. A.A.G. is supported by an NHMRC Emerging Leader 1 Investigator Grant (APP1173784). These funding sources had no role in the design, execution, analysis or reporting of this study.

\section{Compliance with ethical standards}

Conflict of interest The authors declare no competing interests.

Publisher's note Springer Nature remains neutral with regard to jurisdictional claims in published maps and institutional affiliations.

Open Access This article is licensed under a Creative Commons Attribution 4.0 International License, which permits use, sharing, adaptation, distribution and reproduction in any medium or format, as long as you give appropriate credit to the original author(s) and the source, provide a link to the Creative Commons license, and indicate if changes were made. The images or other third party material in this article are included in the article's Creative Commons license, unless indicated otherwise in a credit line to the material. If material is not included in the article's Creative Commons license and your intended use is not permitted by statutory regulation or exceeds the permitted use, you will need to obtain permission directly from the copyright holder. To view a copy of this license, visit http://creativecommons. org/licenses/by/4.0/.

\section{References}

1. Vos T, Allen C, Arora M, Barber RM, Bhutta ZA, Brown A, et al. Global, regional, and national incidence, prevalence, and years lived with disability for 310 diseases and injuries, 1990-2015: a systematic analysis for the Global Burden of Disease Study 2015. Lancet. 2016;388:1545-602.
2. Hunter DJ, Bierma-Zeinstra S. Osteoarthritis. Lancet. 2019; 393:1745-59.

3. Chen A, Gupte C, Akhtar K, Smith P, Cobb J. The global economic cost of osteoarthritis: how the UK compares. arthritis 2012; 2012. https://doi.org/10.1155/2012/698709.

4. Kurtz S, Ong K, Lau E, Mowat F, Halpern M. Projections of primary and revision hip and knee arthroplasty in the United States from 2005 to 2030. J Bone Joint Surg Am. 2007;89:780-5.

5. Culliford D, Maskell J, Judge A, Cooper C, Prieto-Alhambra D, Arden NK. Future projections of total hip and knee arthroplasty in the UK: results from the UK Clinical Practice Research Datalink. Osteoarthritis Cartilage. 2015;23:594-600.

6. Sharif B, Kopec J, Bansback N, Rahman MM, Flanagan WM, Wong $\mathrm{H}$, et al. Projecting the direct cost burden of osteoarthritis in Canada using a microsimulation model. Osteoarthritis Cartilage. 2015;23:1654-63.

7. Nemes S, Gordon M, Rogmark C, Rolfson O. Projections of total hip replacement in Sweden from 2013 to 2030. Acta Orthop. 2014;85:238-43.

8. Hooper G, Lee AJ-J, Rothwell A, Frampton C. Current trends and projections in the utilisation rates of hip and knee replacement in New Zealand from 2001 to 2026. N Z Med J. 2014;127:82-93.

9. Arthritis Australia. Counting the cost-the current and future burden of arthritis. Arthritis Australia; 2016.

10. Ackerman IN, Bohensky MA, Zomer E, Tacey M, Gorelik A, Brand CA, et al. The projected burden of primary total knee and hip replacement for osteoarthritis in Australia to the year 2030. BMC Musculoskelet Disord. 2019;20:90.

11. Ackerman IN, Pratt C, Gorelik A, Liew D. Projected Burden of Osteoarthritis and Rheumatoid Arthritis in Australia: A Population-Level Analysis. Arthritis Care Res. 2018;70:877-83.

12. Salih S, Sutton P. Obesity, knee osteoarthritis and knee arthroplasty: a review. BMC Sports Sci Med Rehabil. 2013;5:25.

13. Zheng H, Chen C. Body mass index and risk of knee osteoarthritis: systematic review and meta-analysis of prospective studies. BMJ Open. 2015;5:e007568.

14. Fehring TK, Odum SM, Griffin WL, Mason JB, McCoy TH. The obesity epidemic: its effect on total joint arthroplasty. J Arthroplasty. 2007;22:71-76.

15. Changulani M, Kalairajah Y, Peel T, Field RE. The relationship between obesity and the age at which hip and knee replacement is undertaken. J Bone Joint Surg Br. 2008;90:360-3.

16. McAlindon TE, Bannuru RR, Sullivan MC, Arden NK, Berenbaum F, Bierma-Zeinstra SM, et al. OARSI guidelines for the non-surgical management of knee osteoarthritis. Osteoarthr Cartil. 2014;22:363-88.

17. Fernandes L, Hagen KB, Bijlsma JWJ, Andreassen O, Christensen $\mathrm{P}$, Conaghan PG, et al. EULAR recommendations for the nonpharmacological core management of hip and knee osteoarthritis. Ann Rheum Dis. 2013;72:1125-1135.

18. Hochberg MC, Altman RD, April KT, Benkhalti M, Guyatt G, McGowan J, et al. American College of Rheumatology 2012 recommendations for the use of nonpharmacologic and pharmacologic therapies in osteoarthritis of the hand, hip, and knee. Arthritis Care Res. 2012;64:465-74.

19. American Academy of Orthopaedic Surgeons. Treatment of osteoarthritis of the knee. Rosemont, IL: American Academy of Orthopaedic Surgeons; 2013.

20. RACGP. Guidelines for the management of knee and hip osteoarthritis. East Melbourne, VIC: Royal Australian College of General Practitioners; 2018

21. Christensen R, Bartels EM, Astrup A, Bliddal H. Effect of weight reduction in obese patients diagnosed with knee osteoarthritis: a systematic review and meta-analysis. Ann Rheum Dis. 2007;66:433-9. 
22. Cohort Profile. The 45 and Up Study. Int J Epidemiol. 2008; 37:941-7.

23. 45 and Up Study Collaborators, Banks E, Redman S, Jorm L, Armstrong B, Bauman A, et al. Cohort profile: the 45 and up study. Int J Epidemiol. 2008;37:941-7.

24. Gollings J. National Joint Replacement Registry Annual Report 2018. Adelaide, Australia: Australian Orthopaedic Association; 2019.

25. Goodman SM, Mandl LA, Mehta B, Navarro-Millan I, Russell LA, Parks ML, et al. Education mitigates the effect of poverty on total knee arthroplasty outcomes. Arthritis Care Res. 2018; 70:884-91.

26. Mota REM, Tarricone R, Ciani O, Bridges JF, Drummond M. Determinants of demand for total hip and knee arthroplasty: a systematic literature review. BMC Health Serv Res. 2012;12:225.

27. Felson DT, Zhang Y. Smoking and osteoarthritis: a review of the evidence and its implications. Osteoarthritis Cartilage. 2015;23:331-3.

28. Fransen M, McConnell S, Harmer AR, Esch MV der, Simic M, Bennell KL Exercise for osteoarthritis of the knee. Cochrane Database Syst Rev. 2015. https://doi.org/10.1002/14651858. CD004376.pub3.

29. Australian Institute of Health and Welfare. The Active Australia Survey: a guide and manual for implementation, analysis and reporting, Table of contents. Canberra, AIHW: 2003.

30. Australian Government Department of Health and Ageing. Australia's physical activity and sedentary behaviour guidelines. Australian Government Department of Health and Ageing; 2014.

31. Pellegrini CA, Ledford G, Hoffman SA, Chang RW, Cameron KA. Preferences and motivation for weight loss among knee replacement patients: implications for a patient-centered weight loss intervention. BMC Musculoskelet Disord. 2017;18. https:// doi.org/10.1186/s12891-017-1687-x.

32. Atukorala I, Makovey J, Lawler L, Messier SP, Bennell K, Hunter DJ. Is there a dose-response relationship between weight loss and symptom improvement in persons with knee osteoarthritis? Arthritis Care Res. 2016;68:1106-14.

33. Cercato C, Fonseca FA. Cardiovascular risk and obesity. Diabetol Metab Syndr. 2019;11. https://doi.org/10.1186/s13098-019-0468-0.

34. Deveza LA, Dai Z, Hunter DJ. The relationship of weight loss to structure modification in knee OA. Osteoarthritis Cartilage. 2019; 0. https://doi.org/10.1016/j.joca.2019.02.794.

35. Teichtahl AJ, Wluka AE, Tanamas SK, Wang Y, Strauss BJ, Proietto $\mathrm{J}$, et al. Weight change and change in tibial cartilage volume and symptoms in obese adults. Ann Rheum Dis. 2015;74:1024-9.
36. Gersing AS, Solka M, Joseph GB, Schwaiger BJ, Heilmeier U, Feuerriegel G, et al. Progression of cartilage degeneration and clinical symptoms in obese and overweight individuals is dependent on the amount of weight loss: 48-month data from the osteoarthritis initiative. Osteoarthr Cartil OARS Osteoarthr Res Soc. 2016;24:1126-34.

37. Hunter DJ, Beavers DP, Eckstein F, Guermazi A, Loeser RF, Nicklas BJ, et al. The Intensive Diet and Exercise for Arthritis (IDEA) trial: 18-month radiographic and MRI outcomes. Osteoarthritis Cartilage. 2015;23:1090-8.

38. Jafarzadeh SR, Clancy M, Li J-S, Apovian CM, Guermazi A, Eckstein $\mathrm{F}$, et al. Changes in the structural features of osteoarthritis in a year of weight loss. Osteoarthritis Cartilage. 2018;26:775-82.

39. Bannuru RR, Osani MC, Vaysbrot EE, Arden NK, Bennell K, Bierma-Zeinstra SMA, et al. OARSI guidelines for the nonsurgical management of knee, hip, and polyarticular osteoarthritis. Osteoarthritis Cartilage. 2019;27:1578-89.

40. Paans N, van den Akker-Scheek I, Dilling RG, Bos M, van der Meer K, Bulstra SK, et al. Effect of exercise and weight loss in people who have hip osteoarthritis and are overweight or obese: a prospective cohort study. Phys Ther. 2013;93:137-46.

41. Reijman M, Pols HaP, Bergink AP, Hazes JMW, Belo JN, Lievense $\mathrm{AM}$, et al. Body mass index associated with onset and progression of osteoarthritis of the knee but not of the hip: the Rotterdam Study. Ann Rheum Dis. 2007;66:158-62.

42. Singer SP, Dammerer D, Krismer M, Liebensteiner MC. Maximum lifetime body mass index is the appropriate predictor of knee and hip osteoarthritis. Arch Orthop Trauma Surg. 2018;138:99-103.

43. Lo T, Parkinson L, Cunich M, Byles J. Discordance between selfreported arthritis and musculoskeletal signs and symptoms in older women. BMC Musculoskelet Disord. 2016;17. https://doi. org/10.1186/s12891-016-1349-4.

44. Kirksey M, Chiu YL, Ma Y, Della Valle AG, Poultsides L, Gerner $\mathrm{P}$, et al. Trends in in-hospital major morbidity and mortality after total joint arthroplasty: United States 1998-2008. Anesth Analg. 2012;115:321-7.

45. Beswick AD, Wylde V, Gooberman-Hill R, Blom A, Dieppe P. What proportion of patients report long-term pain after total hip or knee replacement for osteoarthritis? A systematic review of prospective studies in unselected patients. BMJ Open. 2012;2: e000435.

46. Nevitt MC, Felson DT, Lester G. The osteoarthritis Initiativeprotocol for the cohort study 1: 74. https://oai.epi-ucsf.org/data release/About.asp. 